\title{
The Casey Holter memorial lecture: genetics and embryology of neural tube defects
}

\section{Andrew Copp}

Address: UCL Institute of Child Health, 30 Guilford Street, London WC1N 1EH, UK

Email: Andrew Copp - A.Copp@ich.ucl.ac.uk

from 52nd Annual Meeting of the Society for Research into Hydrocephalus and Spina Bifida Providence, RI, USA. II-I4 June 2008

Published: 3 February 2009

Cerebrospinal Fluid Research 2009, 6(Suppl I):S2 doi:10.1 186/1743-8454-6-SI-S2

This abstract is available from: http://www.cerebrospinalfluidresearch.com/content/6/SI/S2

(c) 2009 Copp; licensee BioMed Central Ltd.

Neurulation is a key event in central nervous system development; in which bending and subsequent fusion of the edges of the neural plate culminate in the formation of the neural tube, the precursor of the brain and spinal cord. Failure of neural tube closure results in neural tube defects (NTDs) in which the lumen of the neural tube remains open to the amniotic fluid environment. Degeneration of the neuroepithelium occurs later in gestation leading to neurological deficit by the time of birth, and severe handicap from the newborn period onwards. While a strong genetic predisposition to human NTDs is generally accepted, the genes that mediate this predisposition have been difficult to identify. In contrast, over 100 genes are known to be required for mouse neural tube closure, by virtue of the NTD phenotypes when they are inactivated in knockout or mutant strains.

The genetic and development events underlying three different types of genetic mouse NTDs can be described as follows. (1) Failure of the initial step of neurulation (socalled Closure 1), leads to the most severe NTD, craniorachischisis, in which the neural tube remains open from midbrain to low spine. Recent studies show that initiation of neurulation requires signalling via the non-canonical Wnt/dishevelled (planar cell polarity) pathway. (2) Disruption of dorso-lateral neural plate bending in the low spinal region, as is seen in the Zic2 mutant mouse, prevents the neural fold tips from coming into apposition, leading to lumbo-sacral spina bifida. Our recent studies demonstrate an essential role for signalling via the BMP and Sonic hedgehog pathways in regulating neural plate bending. (3) Re-opening of the closed neural tube can yield a variety of NTDs, including isolated thoracic spina bifida, not previously seen in mouse genetic models.

Primary prevention of NTDs is now practised clinically using peri-conceptional folic acid therapy. We have demonstrated that folic acid deficiency is also a risk factor for NTDs, but only in the presence of a genetic predisposition, such as seen in the Pax3 (splotch) mutant mouse. However, a proportion of NTDs in humans and mice do not respond to folic acid, and we demonstrated that inositol can be used as an alternative, adjunct therapy where folic acid is ineffective, such as in the Grhl3 (curly tail) mouse mutant. A new clinical trial to evaluate inositol as a preventive agent for NTDs in human pregnancy is now beginning in the UK http://www.pontistudy.ich.ucl.ac.uk. 